\title{
Guardian: A Prototype Intelligent Agent for Intensive-Care Monitoring
}

\author{
Barbara Hayes-Roth ${ }^{1}$ Serdar Uckun ${ }^{1}$ \\ David Gaba $^{2}$ Juliana Barr ${ }^{2}$ \\ Jan Eric Larsson 1 \\ Jane Chien ${ }^{2}$ \\ ${ }^{1}$ Knowledge Systems Laboratory, Stanford University \\ 701 Welch Road B1dg. , Palo Alto, CA 94304 \\ 2 Stanford University School of Medicine and Department of Veterans Affairs \\ 3801 Miranda Ave., Palo Alto, CA 94304 \\ \{hayes-roth, uckun, larsson, gaba\} @KSL.Stanford.Edu
}

\begin{abstract}
A surgical intensive care unit (ICU) is a challenging monitoring environment. The multitude of monitored variables, the high frequency of alarms, and the severity of likely complications and emergencies can overload the cognitive skills of even experienced clinicians. ICU monitoring is also complicated by changes in clinical context. Over the course of a few days, a patient may evolve from a high-vigilance immediate post-operative state to a convalescent state that involves entirely different sets of monitoring principles, problems, and treatments.
\end{abstract}

Guardian is an experimental intelligent agent for monitoring patients in a surgical ICU (Hayes-Roth et al. 1992). Guardian is based on the BB1 blackboard control architecture and is under development in a laboratory environment using simulated and recorded patient data. Guardian has a number of advantages over existing real-time intelligent monitoring architectures. These include multiple reasoning skills, configuration of available knowledge and skills based on context, data reduction based on the availability of computational resources, and dynamic selection of reasoning skills under time pressure. Guardian is composed of a variety of software modules organized in two levels. At the lower level, Guardian has modules that perform data reduction and abstraction tasks. At the higher level, various reasoning skills exist and cooperate under the guidance of BB1. Domain knowledge bases for Guardian are coordinated through a shared ontology for intelligent monitoring and control. These knowledge bases are available to any of Guardian's problem solving components that wish to use them. Incremental knowledge acquisition continues in parallel with the development for Guardian.

This videotape focuses on the dynamic and context-sensitive aspects of reasoning in Guardian. In the demonstration, Guardian starts monitoring a simulated patient who has had open heart surgery and has just been taken from the operating room to the surgical ICU. This situation is defined as the "early postoperative" situation. Later in the demonstration, the patient improves to a more stable situation. Since the domain knowledge bases are extensive, Guardian selects different subsets of problems for which

The Guardian project is sponsored by ARPA/NASA grant NAG2-581 under ARPA order 8607. to prepare short-latency reactions as appropriate for these different contexts. Its choices reflect consideration of several features of known contingencies, such as criticality, side effects, and likelihood - in the given context (Dabija 1994).

During normal operation, over a hundred channels of data flow into Guardian at regular intervals or on demand. A data reduction and dynamic filtering component reduces the incoming data rate based on dynamic attention focus ing decisions and also on the dynamic global rate at which Guardian can process incoming data. A temporal fuzzy pattern recognition component classifies incoming data in terms of clinically-relevant signs and symptoms.

Two component reasoning skills are demonstrated. In the immediate postoperative situation, a reactive diagnosis skill (Ash et al. 1993) utilizes action-based hierarchies to provide short-latency diagnosis and therapeutic response to one of the selected subset of context-relevant clinical problems. Later, a probabilistic causal reasoning skill (Peng \& Reggia 1990) performs associative diagnosis based on clinical signs and symptoms for a non-timestressed problem. These examples illustrate Guardian's ability to make runtime choices among alternative reasoning methods based on problem characteristics and the availability of data, knowledge, and real-time computa tional resources.

\section{References}

Hayes-Roth, B., et al. 1992. Guardian: a prototype intelligent agent for intensive-care monitoring. Artificial Intelligence in Medicine 4 (2): 165-185.

Dabija, V. 1994. Deciding whether to plan to react. Ph.D. diss., Dept. of Computer Science, Stanford University.

Ash, D.; Gold, G.; Seiver, A.; and Hayes-Roth, B. 1993. Guaranteeing real-time performance with limited resources. Artificial Intelligence in Medicine 5(1): 49-66.

Peng, Y.; and Reggia, J. 1990. Abductive inference models for diagnostic problem-solving. New York, NY: Springer-Verlag. 